\title{
Non-Contact Force Measurement for Current Collection in a 25kV Overhead Line Electrified Railway
}

\author{
Dr M.R. Arthington ${ }^{1}$, P.T. Barnes ${ }^{2}$, Professor S.R. Duncan ${ }^{3}$ \\ ${ }^{1}$ Matthew Arthington, Oxford University, United Kingdom, \\ ${ }^{2}$ Paul Barnes, Network Rail, paul.barnes@networkrail.co.uk \\ ${ }^{3}$ Stephen Duncan, Oxford University United Kingdom
}

Keywords: Overhead Line Electrification, Force Measurement, Image processing

\begin{abstract}
Network Rail's Western Route is undergoing a wide-ranging modernisation programme, with $£ 7.5$ bn investment in 175 miles of electrification and infrastructure upgrades allowing the introduction of four new fleets of train by 2019. This paper reviews the potential utilisation of camera imagery to inform maintenance processes that need to absorb a step change in pantograph movements and OLE management.
\end{abstract}

\section{Introduction}

\subsection{Background}

In 2009, the UK Government announced the electrification of the Western route from London to Bristol and Swansea. A schematic of the route is shown - solid lines are the electrified routes

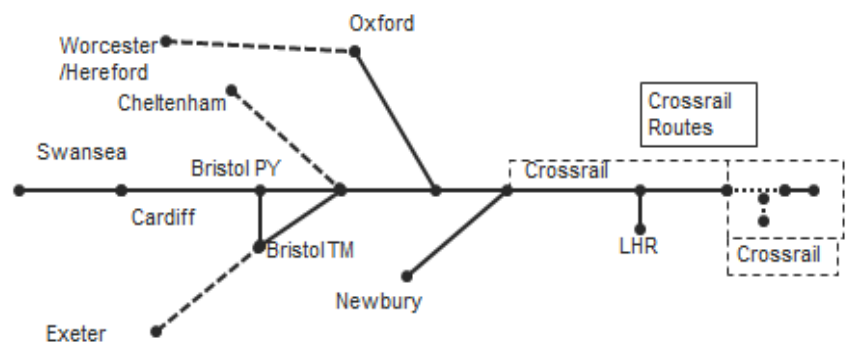

Figure 1: Schematic of Western \& Wales IEP route

This will be the first major rail electrification project since the 1980's, and twenty years on from the 15 electrified route miles between Paddington \& Heathrow Airport, which has used a dedicated fleet of Siemens Class 332 Heathrow Express Units, with HS-A pantographs operating at $100 \mathrm{mph}$, and in later years mix of 332 and Siemens Class 360 units (Heathrow Connect) with HAS Mk 1 pantographs.

In parallel, the Department for Transport had developed a strategy to replace the ageing HST diesel fleet, which has been the backbone of the $125 \mathrm{mph}$ Western route operation for over 30 years. Preferred bidder status was awarded to Agility Trains in 2009 with the Hitachi Super Express train as the replacement. The IEP fleet will use an HSX pantograph developed by Brecknell Willis. This creates a new system configuration undergoing live dynamic testing in 2016 following software modelling in the design stage. In terms of system design, the IEP fleet is required to operate in multiple pan formation, normally $2 \times 5$ cars at $125 \mathrm{mph}-\mathrm{a}$ first in the UK.

In 2008, the Crossrail bill became an Act of Parliament clearing the way for the construction and implementation of a new service from Shenfield \& Abbey Wood to Reading and Heathrow. This introduces a new fleet of Bombardier Class 345 units with two HSP Mk 2 pans at speeds up to $90 \mathrm{mph}$.

In order to utilise the OHLE system for suburban services, the current Operator GWR is introducing Siemens Class 387 units - capable of $110 \mathrm{mph}$ and with up to three HSP pans. These will replace the existing 20 year old diesel turbo fleets

The fourth new fleet of Hitachi AT300 trains was announced by GWR in March 2015. These are a variant on the bi-mode IEP with the same pantograph configuration for OLE but more powerful diesels for the steep gradients west of Exeter.

\subsection{Western electrified route by 2019}

The introduction of the new fleets creates a step change in use of the OHL system from Paddington to Heathrow Airport Junction - and a complete new system beyond there.

\section{Context: Pan Pass Stress Map}

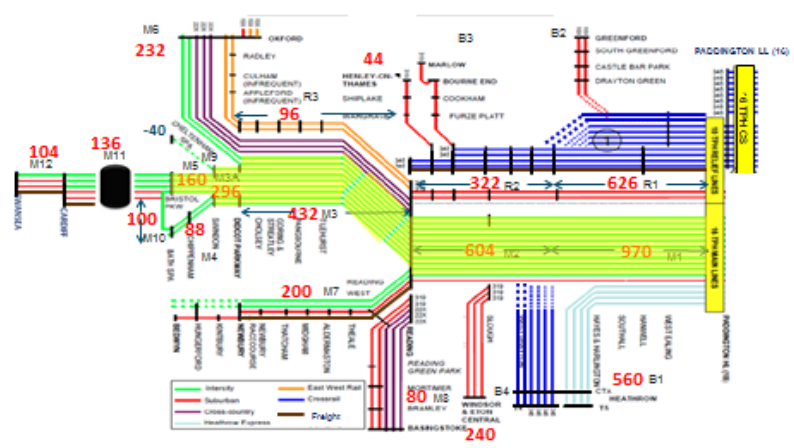

Figure 2: Schematic of Pantograph passages 
An indication of the increased importance of OLE monitoring with the new timetable is demonstrated by a five-fold increase in the number of pan passages averaged to a single wire between Paddington and [Heathrow] Airport Junction.

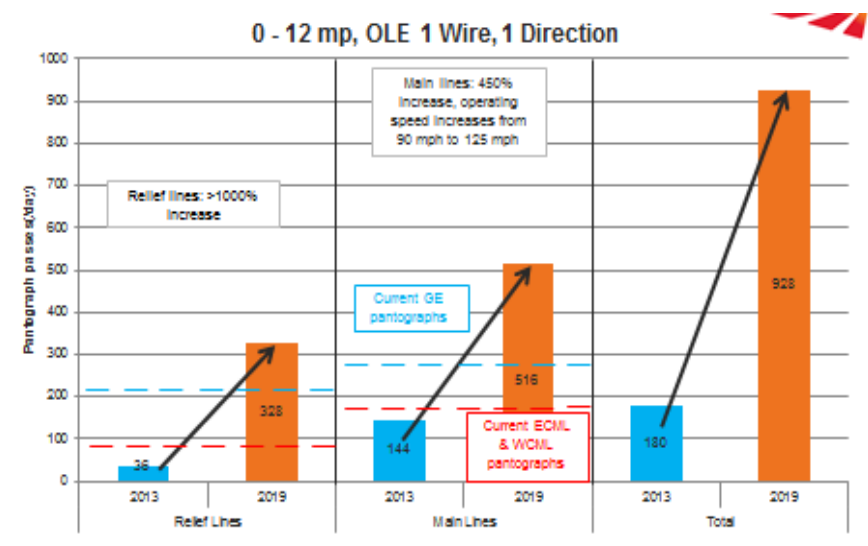

Figure 3: Step change in use - example 0-12 MP

The maintenance challenge is increased because from a relatively simple system of two pan types only in single pan formation, we move to at least five pan types with a mix of single and multiple pan operation. It will be the only route where multiple pan trains operate at $125 \mathrm{mph}$ - leading to an interesting dynamic to explore both on the existing section and the Series 1 sections beyond Airport Junction

\section{OHLE}

\subsection{Series 1 Requirements}

The three main Overhead Line Systems in Network Rail use are the Mark 3, UK1 and Series 2, each designed to meet an electrification scheme. Only Series 2 is amended to be TSI compliant, but none have been designed for or used continuously with multiple pantograph operation at $125 \mathrm{mph}$. Therefore rather than redesign existing systems, the decision was made to design a new overhead line system with principal requirements of:

- TSI compliance;

- Nominal fault current rating of $12 \mathrm{kA}$.

- Traction power of $25 \mathrm{kV} 50 \mathrm{~Hz}$ single phase AC supply, Classic or A.T.

- Line speed capability up to $140 \mathrm{mph}$.

- Design ambient operating temperature range of

- $18^{\circ} \mathrm{C}$ to $+40^{\circ} \mathrm{C}$.

- Compatible with existing / future UK pantograph configurations.
In addition a Network Rail team identified what became known as the "97 problem statements" in order to inform improvements in the design. These are summarised as:

- Poor reliability of electrical connections generally and mechanical system at higher speeds

- Poor maintainability with complex supply chain

- Ambiguous allocation design rules

- Not tolerant of extreme weather or seasonal effects

- Relatively large envelope of "Live" equipment

The outcome is the design known as Series 1, based on a Furrer+Frey FL200 / 260 \& GEFF design with an Improved reliability (MTBSAF <1 failure per 250 years / STkm) and less maintenance (20\% fewer cat A OLE staff). It has met the requirements with a $16.5 \mathrm{kN}$ tension - higher than elsewhere in the UK...

\subsection{Typical design challenges in Western Route}

The design of OHLE requires creativity in architecture and science to balance the system requirements of the railway for a constant tension with the clearances required and aesthetics. Western route use a gradient model to support designs and, as the Henley branch demonstrates, how the juxtaposition of Level Crossings and Overstructures challenges gradients as an example. The range here pre-design was from $<1$ in 1000 to 1 in 250 (compromised). The ability of the pantograph to maintain contact and design force and collect current in line with standards is challenged even at low speeds.

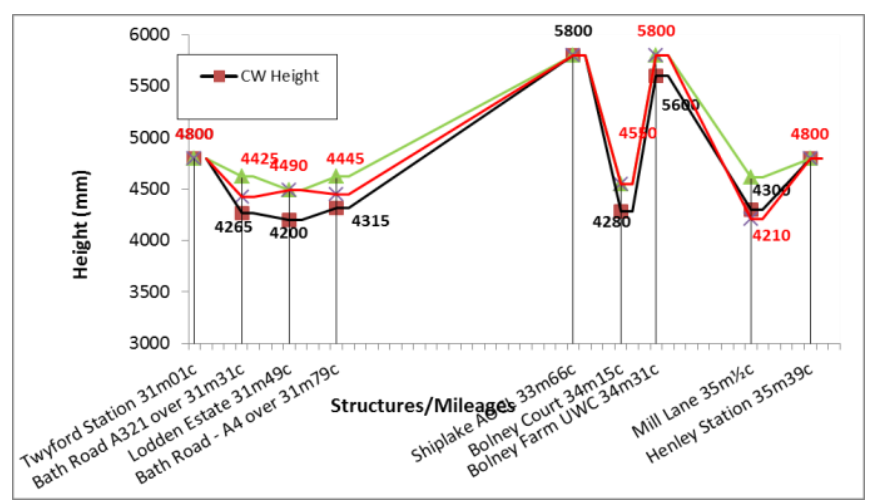

Figure 4: Gradients model - gradients Henley Branch

The measurement of force and particularly longitudinal acceleration is seen as a key indicator for failure modes associated with dewirement. The models help us to identify our gradient changes and thus we seek to programme data collection to changes in gradient where we might anticipate trigger points. We can then align data with other parameters such as wire wear and changes in registration to identify degradation curves in the subsystem. 


\section{Approach and process}

\subsection{Approach}

A key operational requirement is to maintain the required contact force between the pantograph and the overhead line. At present, the contact force is measured using an instrumented pantograph mounted on a specialist measurement train. The approach described in this paper uses image processing to extract the vertical movement of the pantograph from images taken using existing on-board cameras, and the contact force between the pantograph and the overhead line can then be estimated.

Optical measurement systems offer several inherent advantages over contacting systems, including electrical isolation, zero additional mass in dynamic systems and a potentially wide breadth of measurements in a single sensor. Machine vision [1] has been applied in a wide variety of industries, from production lines to self-driving cars, in order to measure and monitor processes. In the rail industry, machine vision is currently used in plain line pattern recognition (PLPR) for monitoring wear in rails and is also implemented in systems in Japan and Taiwan for monitoring OLE using multiple cameras and strong illumination.

We are using existing camera systems, initially installed for monitoring only, to acquire measurements of the pantograph height. Using the height data and the dynamic model of the pantograph system we then estimate the force developed on the carbon strip.

In future, many more trains running on UK track will have cameras installed to monitor the pantograph and installing markers and lighting would allow all of these cameras to become intelligent OLE monitors too.

\subsection{Process - Machine Vision for Pantograph Height}

Two retroreflective markers are applied to the outer horns of the pantograph, as shown in Figure 4. These markers are visible in every video frame where the pantograph is in contact with the wire. They permit the use of relatively lowpower lights to highlight the markers in daylight and nighttime conditions.

In normal pantograph motion, the markers follow a vertical trajectory. To find the position of the bright marker spots, the maximum brightness positions within the regions of interest are found. Constraints on the width of the markers and the need for both to move in near unison prevent spurious reflections being mistaken for markers. The regions of interest (shown in Figure 5) are identified during calibration.
The camera and the pantograph are calibrated offline. This permits the location of a bright marker spot in a video frame to be transformed into the height of the pantograph at that point. Using two markers also permits the roll of the pantograph to be estimated. Once the height and roll data for the pantograph head are found in every frame, this is output as a time series to be matched with the train location time series. The height position is used by the contact-force estimation algorithm.

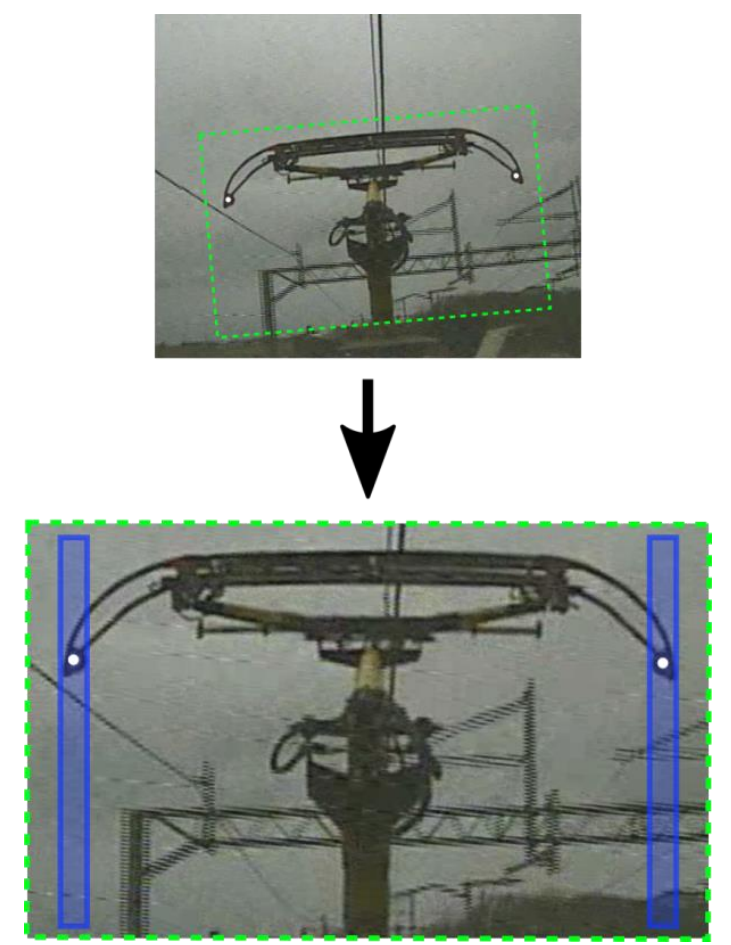

Figure 5: This image shows the marked pantograph in the raw video footage and the regions in which the retroreflective markers will appear in the image. Markers are identified by their brightness and their approximately equal height.

If the resolution of the camera is sufficient, the width of the wires in the view may also be estimated. This allows the differentiation between contact wires and catenary wires to be made, and the contact position between the pantograph and the contact wire to be measured. If the stagger of the contact wire is too great, a flag may be raised for further monitoring of that section.

\subsection{Force Estimation}

A dynamic model of the pantograph can be used to determine the acceleration of the pantograph head in response to a change in the contact force between the pantograph and the overhead line, and the acceleration can then be integrated twice to give the variation in the vertical position of the pantograph. However, this model can be used in reverse: 
given a measurement of pantograph height then this can be used to estimate the variation in contact force. Deriving an estimate of the contact force by simply inverting the model is known to be sensitive to measurement errors and sensor noise, so the force estimate is obtained using a Kalman filter which provides an optimal estimate of the contact force in the presence of noise. Figure 6 compares an estimate of the contact force with the actual force, using data obtained from a simulation of the OLE dynamics. The Kalman filter allows for the aerodynamic effects on the pantograph, which depend upon train speed.

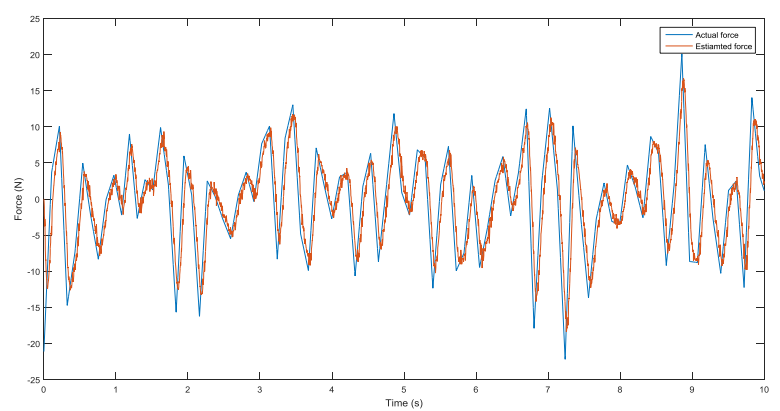

Figure 6 - Actual force (blue) and estimate of force (red) obtained from Kalman filter applied to measurement of vertical position of pantograph from simulation of train running at $120 \mathrm{kph}$.

\section{Development Trials}

A Virgin Trains trial has begun to investigate the potential for tracking an unmarked pantograph to monitor wire stagger, contact conditions and dropper connections. Semi-automation of the fault-detection process will be achieved to reduce the workload on engineers.

The HEX trial uses a marked pantograph to achieve an accurate and robust method for pantograph tracking, and therefore reliable vertical force estimation.

Large volumes of video data are created by these systems, and recovering the force on the pantograph and tracking wire positions has the potential to reduce the volume of useful data that needs to be stored, and certainly increases the speed at which pertinent information about the state of the OLE can be delivered to the necessary recipients.

This work goes part of the way towards achieving the requirements of BS EN 50317[2] by measuring vertical contact force to monitor correct current collection. The sampling frequency of force measurement may not be sufficient to meet the standard, but it is sufficient to detect deviations from nominal force, which is the most important aspect of the monitoring system from a maintenance perspective. The added benefit that this monitoring can be performed daily more than makes up for the lack of temporal resolution in the force data.

\section{Benefit Realisation}

The key business benefit is to reduce the number of OLE related incidents which create unplanned outage on the system. This will contribute to train service punctuality and availability as shown in the system models. Less accurate, more frequent monitoring offers a readily available solution to acquiring OLE contact force measurement. This information is vital for implementing a well-informed riskbased maintenance strategy. The graph below is reproduced from system models described in [3]. It shows the significant effect of short circuit faults in reducing the Public Performance Measure (PPM, the industry standard for train punctuality

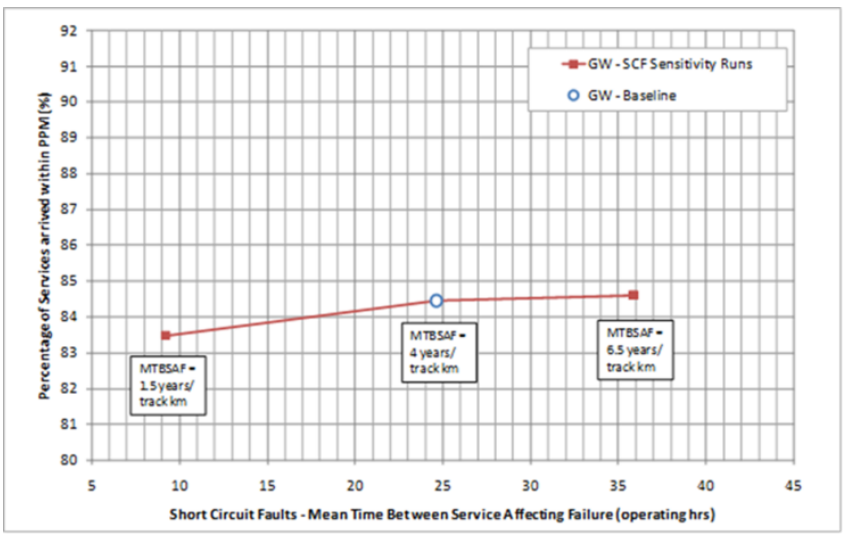

Figure 7 - Sensitivity of punctuality (PPM) and OLE short circuit fault, from [3].

Less accurate, more frequent monitoring offers a readily available solution for identifying changes in the OLE system through which we can predict and prevent short circuit faults and support punctuality. Such information is vital for implementing a well-informed risk-based maintenance strategy. The method outlined here provides a rapid and readily available technique for obtaining data from a range of pantograph types. It perfectly complements data obtained from conventionally instrumented pantographs while offering the opportunity to collect data much more frequently than is presently done. This type of data is exactly the sort needed for big data analysis of networks,

\section{Acknowledgements}

The authors are grateful for the technical support and advice of Rail Industry colleagues particularly Glenn Wiles and Andy Mackintosh, and train operators, particularly Virgin Trains and Heathrow Express Ltd. 


\section{References}

[1] R. Szeliski, "Computer vision: algorithms and applications", Springer Science \& Business Media, (2010)

[2] BSi, "BS EN 50317 Railway applications - Current collection systems - Requirements for and validation of measurements of the dynamic interaction between pantograph and overhead contact line”, BSi (2012).

[3] Barnes PT, Tunley, DR, Gallop MC, "Systems modelling of Network Rail's Western Route", IET/IAM "The Asset Management Conference", London, (2015) 
